\title{
Epigenetic Regulations of GABAergic Neurotransmission: Relevance for Neurological Disorders and Epigenetic Therapy
}

\author{
Shikshya Shrestha Steven M. Offer \\ Mayo Graduate School, Mayo Clinic College of Medicine, Rochester, Minn., USA
}

Key Words

GABA · GABAergic neurotransmission · Neurological disorders · DNA methylation · Histone modification

\begin{abstract}
The GABAergic neurotransmission is a highly conserved system that has been attributed to various regulatory events. There has been a notable number of studies on the importance of GABAergic neurotransmission, both excitatory and inhibitory, in neurogenesis and central nervous system development including its control of neuronal cell proliferation and migration, synaptogenesis, dendrite formation and branching, and new neuronal cell integration in the adult brain. There has been remarkable progress in understanding the epigenetic regulations of GABAergic genes and their aberrant expressions in various neurological disorders such as autism spectrum disorder, Rett's syndrome, schizophrenia and PWS. The roles of histone modifications, chromatin looping and gene methylation have been implicated in altered regulations of key genes in the GABAergic pathway. Taken together, they affect the functioning of GABAergic neurotransmission and disrupt various events in brain development. Here, we focus on the role of GABAergic neurotransmission in brain development and on how various genetic and epigenetic events regulate the GABAergic genes in pre- and postnatal brain. We also discuss how these regulatory mechanisms contribute to the pathogenesis of neurological disorders and, therefore, can be used in the development of potential epigenetic therapy for these diseases.




\section{Introduction}

$\gamma$-Aminobutyric acid (GABA) is a nonprotein amino acid that is a major inhibitory neurotransmitter for fast synaptic transmission in the adult brain. It is also fairly abundant and widespread in early embryonic life, and has excitatory synaptic transmission in the immature brain [1]. GABA neurotransmission is a highly conserved phenomenon present in nearly all organisms, ranging from bacteria to humans [2]. The pathway of GABAergic neurotransmission is schematically represented in figure 1. GABAergic neurons synthesize GABA from L-glutamate, and the reaction is catalyzed by an enzyme glutamic acid decarboxylase (GAD; fig. 1a) [3, 4]. There are two isoforms of GAD in mammals (GAD67, MW = $67 \mathrm{kDa}$, and GAD65, MW $=65 \mathrm{kDa}$ ) that are encoded by two genes on different chromosomes - GAD1 and GAD2 [3]. GAD1 and GAD2 map to chromosome 2q.31.1 and 10p11.23, respectively [5]. Once produced, GABA is stored in synaptic vesicles of GABAergic neurons facilitated by vesicular GABA transporter (VGAT) and undergoes calcium ion-dependent release upon depolarization (fig. 1a). The GABA release from presynaptic terminals is followed by the actions of GABA receptors $\left(\mathrm{GABA}_{\mathrm{A}}\right.$ and $\left.G A B A_{B}\right)$, which mediate GABAergic signaling, via specific actions and neurotransmission of GABA, by using a class of GABA transporters (GATs) [6, 7]. GABA $\mathrm{A}_{\mathrm{A}}$ receptors are ion channels, while $\mathrm{GABA}_{\mathrm{B}}$ receptors are G-protein-coupled receptors. Stimulation of $\mathrm{GABA}_{\mathrm{A}}$ receptor increased permeability to chloride ions that results in inhibitory or hyperpolarizing effect by increasing the postsynaptic membrane potential (fig. 1b). Stimulation of $\mathrm{GABA}_{\mathrm{B}}$ receptor modulates cyclic adenosine monophosphate (CAMP) production and also increases the potassium conductance and results in hyperpolarization or inhibits voltage-gated calcium channels (fig. 1d) $[3,4] . G A B A_{A}$ receptor also mediates the reversal potential or the depolarizing effect (fig. 1c) in immature brain, when there is decreased expression of $\mathrm{K}-\mathrm{Cl}$ cotransporter 2 (KCC2; outward $\mathrm{Cl}^{-}$channel) and increased expression of Na-K-Cl cotransporter 1 (NKCC1; inward $\mathrm{Cl}^{-}$channel) [8-11]. GATs, located in pre- and postsynaptic terminals, then terminate phasic GABAergic neurotransmission and also recycle GABA for reuse (fig. 1a) [12].

Multiple functions of GABA have evolved since its discovery. As discussed in a later section of this review, GABA plays a crucial role in controlling both neuronal inhibitory and excitatory states, as well as in wide range of developmental and behavioral patterns in the brain. Alterations in GABA and GABAergic signaling have been established as an etiology of various neurological disorders, such as autism spectrum disorder (ASD), Rett's syndrome, Prader-Willi Syndrome (PWS) and schizophrenia (SZ). Studies on the pathogenesis of these disorders have shed light on not only genetic but also epigenetic regulations of various GABAergic genes. GABAerigic neurogenesis itself undergoes regulation of various transcription factors (distal-less, Dlx; Pfta1; Tal; globin transcription factor, GATA) in the prenatal brain. In the postnatal brain, genetic polymorphisms have been reported in some GABAergic genes. Additionally, there are epigenetic markers including histone methylation, acetylation, gene methylation and chromatin loop formations regulating various GABAergic genes. The primary aim of this review is to provide a detailed overview of epigenetic regulations of GABAergic neurotransmission in order to understand the relevance of its epigenetic marks on the pathogenesis of various neurological disorders. We first present a background on the GABA neurotransmitter and its role in the postnatal brain development. Then, we discuss the genetic and epigenetic regulations in important GABAergic genes and how alterations on these marks can affect the expression of GABAergic genes. On a final note, we explore the bench-to-bedside translational aspects of these modifications in discovering potential epigenetic therapies for neurological disorders. 


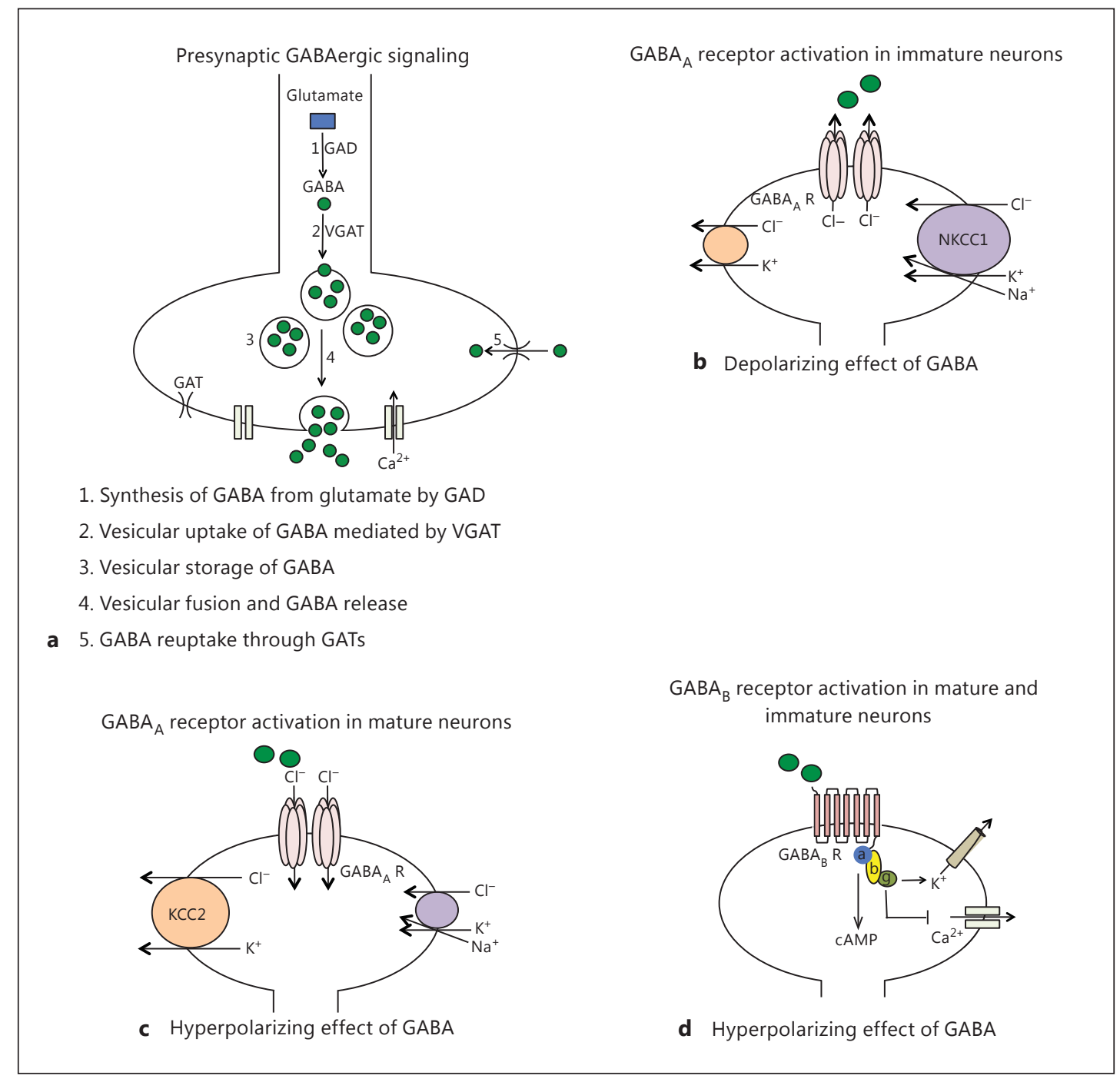

Fig. 1. a Presynaptic GABA signaling. GABAergic neurons synthesize GABA in the cytoplasm by the action of GAD. GABA is then transported into the synaptic vesicle by VGAT. GABA release activates ligand-gated $\mathrm{GABA}_{\mathrm{A}}$ and $\mathrm{G}$-coupled $\mathrm{GABA}_{\mathrm{B}}$ receptors resulting in hyperpolarizing effects by increasing postsynaptic membrane potential. $\mathbf{b} \mathrm{GABA}_{\mathrm{A}}$ receptor depolarizing effect. $\mathrm{GABA}_{\mathrm{A}}$ receptor polarity is converse in immature neurons, in which the expression of NKCC1 is increased and KCC2 is decreased resulting in higher intracellular $\mathrm{Cl}^{-}$-induced depolarizing effect. $\mathrm{c} \mathrm{GABA}_{\mathrm{A}}$ receptor hyperpolarizing effect. $\mathrm{GABA}_{\mathrm{A}}$ receptors in mature neurons have an increased expression of $\mathrm{KCC} 2$, outward $\mathrm{Cl}^{-}$channel, which results in lower intracellular $\mathrm{Cl}^{-}$and hyperpolarizing effect. $\mathbf{d} \mathrm{GABA}_{\mathrm{B}}$ receptor signaling. $\mathrm{GABA}_{\mathrm{B}}$ receptor, on the other hand, modulates cAMP production and also increases the potassium conductance and results in hyperpolarization or inhibits voltagegated calcium channel [13, 14, 23-27].

\section{Discovery of GABA - Both Inhibitory and Excitatory Neurotransmitter}

The discovery of the GABA neurotransmitter, which accounts for almost half of synaptic activity in the brain, dates back to 1950s from the works of Eugene Roberts on mouse neuroblastoma [13]. Since its discovery, there has been a plethora of evidence demonstrating the inhibitory neurotransmission or hyperpolarizing function of GABA in different regions of the 
adult brain (fig. 1b) [14]. For instance, intracellular recordings from human neocortical slices in vitro resulted in inhibitory postsynaptic potential (IPSP) [15], rat cortical neurons conferring sensitivity to GABA exhibited IPSP [16] and inferior colliculus neurons when exposed to GABA caused inhibition of the spontaneous firing of the neurons [17]. In general, GABA was demonstrated to have an inhibitory effect in neuronal cells in vertebrates and invertebrates [2,15-20] (fig. 1c). The opposing effect of GABA as an excitatory neurotransmitter or depolarizing factor is prevalent in the immature brain, starting from the embryonic period to the beginning of postnatal life. This has been demonstrated by studies of hippocampal slices from young vertebrates (rat, rabbit and kitten) showing predominantly excitatory synaptic events [21-24]. Patch recordings of rat CA3 pyramidal cells demonstrated that the developmental switch in GABA signaling from excitatory to inhibitory neurotransmitter occurs at around postnatal days P8-P10 [25]. Studies on immature neurons show membrane depolarization and GABA developmental switch upon $\mathrm{GABA}_{\mathrm{A}}$ activation mediated by two major intracellular $\mathrm{Cl}^{-}$transporters: the inward flow of $\mathrm{Cl}^{-}$ions is regulated by the NKCC1 and the outward flow by the KCC2 [8-11]. NKCC1 has a low expression in the rat hippocampus at birth, but shows an increasing expression in brain regions during early development, starting at the end of the first postnatal week; on the other hand, KCC2 show delayed expression in these brain regions [8-11]. The GABA developmental switch is an important step in the GABAergic neurotransmission. Optimal balance between excitatory and inhibitory GABA neurotransmission plays an important role in GABA signaling contributing to proper neurodevelopment, starting from early circuit formation to adult neurogenesis.

\section{GABAergic Neurotransmission - Signaling and Role in Postnatal Brain Development}

GABAergic neurotransmission, early (postnatal/immature brain) and late (in adult brain), has been evolutionally conserved, so it may be a general feature of brain development. GABAergic neurotransmission plays a role in the proliferation, migration and integration of other neuronal progenitors that are crucial for the modulation of brain patterns, and confers various trophic functions and contributes to synaptic plasticity [26]. The early excitatory property of GABAergic signaling in the immature brain generates giant depolarizing potentials (GDPs) that play a role in synchronized circuit formation and maintains synergy with other neurotransmitters, including glutamate and $N$-methyl-D-aspartate (NMDA) [24, 27, 28]. GABA neurotransmission via $\mathrm{GABA}_{\mathrm{A}}$ and $\mathrm{GABA}_{\mathrm{B}}$ receptors controls excess proliferation of neuronal stem cells in the postnatal/adult subventricular zone and adult hippocampus, respectively, and influences DNA synthesis $[2,29,30]$. In vitro experiments on embryonic stem cells and neural crest stem cells showed that induced endogenous $\mathrm{GABA}_{\mathrm{A}}$ receptor activation decreases cell numbers and mitotic events in embryonic stem and neural crest stem cells by negatively regulating S-phase cell cycle progression in a p-H2AX-dependent manner [31]. GABA neurotransmission promotes neuronal migration out of the ventricular zone, consequently leading to cortical maturation [2, 29, 32]. Additionally, the trophic action of GABA signaling contributes to dendrite outgrowth and synapse formation in a $\mathrm{Ca}^{2+}$ influx and $\mathrm{Ca}^{2+}$-dependent kinase activation-dependent manner [33]. It has been established that GABA operate before glutamate receptors and synapse formation, so it is not surprising that GABAergic neurotransmission plays a role in synapse communication [33]. The excitatory effect of GABAergic neurotransmission makes GABA an ideal regulator for synaptogenesis. GABAergic neurotransmission allows for excitatory synapse formation owing to its high cytoplasmic chloride content [34, 35]. Unlike glutamatergic signaling, GABAergic neurotransmission also exerts a 'shunting inhibition' by keeping the synaptic potential identical to the 
$\mathrm{Cl}^{-}$reversal potential. This helps prevent the long-lasting activation of voltage-gated $\mathrm{Ca}^{2+}$ channels and inhibits $\mathrm{Ca}^{2+}$ excitotoxicity [34].Wang and Kriegstein [36], using patch recording, demonstrated NMDA receptor activation due to the GABA depolarizing effect that also regulated excitatory synapse formation. This phenomenon of synaptogenesis is not only important in the postnatal neuron but also in the synaptic integration of new neurons formed during adult neurogenesis. Newly generated neuronal cells in adult brain utilization of GABAergic depolarizing neurotransmission precedes glutamatergic excitation in order to regulate dendritic length and branching [35]. Furthermore, GABAergic depolarizing neurotransmission relays a feedback loop and itself promotes the GABAergic developmental switch from excitation to inhibition; the mechanism behind the phenomenon is the increased transcription of KCC2 (outward $\mathrm{Cl}^{-}$channel) mRNA by GABA-mediated depolarization [25]. All these observations indicate that GABAergic neurotransmission has many facets of control of brain developmental stages - from early neuronal shape sculpting to adult neurogenesis, functional balance and architecture of neural circuits, as well as transcriptional regulation.

Disruptions in the GABAergic neurotransmission most certainly impede sequential events in brain development, which have been demonstrated in various in vitro and in vivo studies through the use of various receptor agonists or antagonists and mice knockout (KO) models. For instance, GABA-dependent regulation of neural crest stem cell proliferation is reversed in the presence of GABA receptor antagonist or in GABA receptor null mice [2, 29-31]. GABA signaling-dependent inhibition of neuronal migration is achieved by direct blockade of GABA receptors using receptor antagonist or inhibitors, or by indirect modulation of downstream pathways including blockade of NKCC1 or in the NKCC1-KO mouse model [32]. Likewise, dendritic growth and structural integrity via GABA-depolarizing neurotransmission was shown to improve in the presence of $\mathrm{GABA}_{\mathrm{A}}$ receptor agonist and decline in NKCC1-knockdown cells [35]. GABA-mediated developmental switch or transformation of neurons from a depolarizing to a hyperpolarizing state is inhibited via chronic blockade of $\mathrm{GABA}_{\mathrm{A}}$ receptors using the receptor antagonist; this also significantly decreased the transcription of KCC2 mRNA [25]. These observations are also complemented by various in vitro, ex vivo and in vivo studies on the association of GABAergic neurotransmission deregulations with various forms of intellectual and cognitive disorders such as ASD, Rett syndrome, PWS, SZ and others [9, 37-40].

\section{Genetic Regulation of Pre- and Post-GABAergic Neurotransmission}

GABAergic neurons are expressed throughout the central nervous system (CNS) in varying levels, more prominently in the corticolimbic circuit region (cerebral cortex and hippocampus) $[29,41]$. The origination and morphogenesis of GABAergic neurons from its precursor cells, as well as the roles of GABAergic neurons and signaling in sequential events of neurogenesis, from prenatal to adult brain development have been the focus of multiple reviews to date. GABAergic neuron development is a highly regulated mechanism. The GABAergic neuron formation from neuroepithelial GABAergic progenitor populations and GABA fate determination (excitatory vs. inhibitory) occur in all compartments of the neural tube: forebrain (telencephalon and diencephalon), midbrain and hindbrain (fig. 2) [42, 43]. Achim et al. [42] comprehensively reviewed the regulation of GABAergic neurogenesis across the CNS (telencephalon-anterior diencephalon, posterior diencephalon-midbrain and hindbrain-spinal cord); their article discusses the discrete gene expression patterns and transcription factor activation in different brain regions controlling the GABAergic neurons and signaling. Briefly, Dlx family gene expressions are hallmarks of GABAergic precursors in telencephalon-anterior diencephalon, Gata2 and T-cell acute lymphocytic leukemia-2 (Tal2) 
Shrestha and Offer: Epigenetic Regulations of GABAergic Neurotransmission:

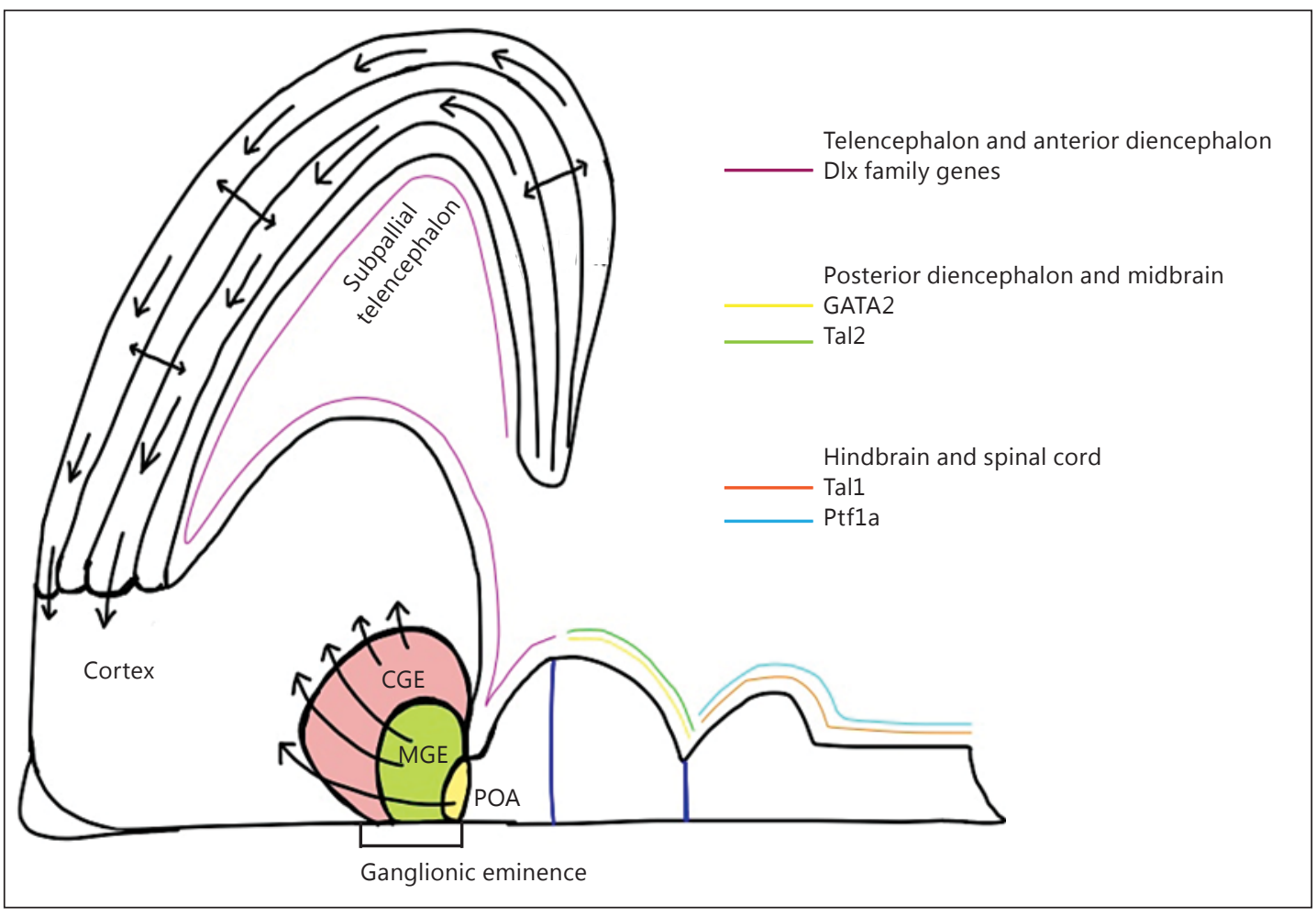

Fig. 2. Hallmark gene expressions control GABAergic neurogenesis and migration. Transcription factors regulating GABAergic gene neurogenesis in different regions of the CNS represented by colored lines - telencephalon-anterior diencephalon (Dlx family genes), posterior diencephalon-midbrain (Gata2 and Tal2) and hindbrain-spinal cord (Tal1 and Ptf1a). Tangential migration of GABAegic neurons occurs from the pallial telencephalon and ganglionic eminence to the cortical region of the brain, represented by black arrows. $\mathrm{CGE}=$ Caudal ganglionic eminence; $\mathrm{MGE}=$ medial ganglionic eminence; $\mathrm{POA}=$ preoptic area. Adapted from Achim et al. [42] and Sultan et al. [44].

in posterior diencephalon-midbrain, and Tal1 and phytochrome and flowering time 1 (Ptf1a) in hindbrain-spinal cord (fig. 2). Each of these genes carries out major functions in GABAergic neuron development, including generation, differentiation, growth, tangential migration (into the cortex and hippocampus originating from the subpallial telencephalon and ganglionic eminence; fig. 2) and fate determination of GABAergic neurons in respective brain regions [42-44]. Differential expression of NKCC1 and KCC2 is involved in controlling GABAergic neuronal potential and developmental switch [8-11]. Transforming growth factor- $\beta$ (TGF- $\beta$ ) signaling proteins also co-express and interact with Dlx transcription factors and regulate the GABAergic neuron development in the telencephalic region [45]. Additionally, various neurotropic factors - brain-derived neurotrophic factor (BDNF), glial cell-derived neurotrophic factor and neurotrophin-4 - are required for GABAergic neuronal migration [42, 46].

Dlx genes, encoding for homeodomain transcription factors, are therefore involved in the regulation of GABA and GABAergic signaling via direct action or via transcription factor activation [42, 47]. Genetic polymorphisms in these genes affect prenatal GABAergic neuron differentiation and migration. One study showed the loss of glutamic acid decarboxylase 1 (Gad1) expression in the olfactory bulb and ventral telencephalon of $D l \times 1^{-/-} / D l \times 2^{-/-}$mice, where Gad1 expression was downstream of Dlx1 and Dlx2 expression, and the phenotype was rescued with the ectopic expression of the Dlx genes in mice telencephalon slice cultures [47]. 
In addition to transcriptional regulation by the Dlx genes, several polymorphisms have been reported in the DLX1, 2, 5 and 6 genes in autistic patients that interfere with normal longevity and differentiation of GABAergic interneurons [48-50]. Likewise, Gad1 is also a downstream target of Pft1a. Hence, in prenatal brain, transcription factors including the Dlx family, GATA2, $\mathrm{Pft} 1 \mathrm{a}$ and Tal1/2 are involved in the maintenance of GABAergic neuron formation, migration and neuron-specific gene expression via direct action or by regulation of other GABAergic genes [42].

Following neurogenesis and tangential migration, GABAergic neurons form a chief component of corticolimbic circuitry $[29,51]$. These neurons demonstrate heterogeneity in terms of gene expression markers depending on the location of these neurons in the CNS; however, these neurons have a general gene expression signature necessary for GABA neurotransmitter metabolism and signaling, including glutamate decarboxylase or GAD and GATs encoding genes $[41,52]$. These hallmark GABAergic genes undergo genetic modifications, including single nucleotide polymorphisms (SNPs). A family-based study on 235 Chinese Han family trios looking at the association between the GAD1 gene and SZ patients using comprehensive genomic mapping of the GAD1 gene showed preferential transmission of an SNP (rs3791878-G allele) and a haplotype (rs3762556-C|rs3791878-G|rs6755102-C) of the GAD1 gene [53]. There are also reported mutations in $\mathrm{GABA}_{\mathrm{A}}$ receptor subunit genes that are associated with genetic epilepsy syndromes, including $\mathrm{GABRA}_{1}(\mathrm{~A} 322 \mathrm{D}), \mathrm{GABRG}_{2}$ (R43Q), GABRG 2 (K289M) and $\mathrm{GABRG}_{2}(\mathrm{R} 82 \mathrm{Q})[54,55]$. An analysis of $\mathrm{GABRG}_{2}(\mathrm{~K} 289 \mathrm{M})$ using wild-type and mutated Xenopus laevis oocytes showed decreased amplitudes of GABAactivated currents [55]. Some, but not all, of the additional variations in these genes referenced in the dbSNP and ClinVar databases as pathogenic include GAD1 (p.Ser12Cys), GABRA (p.Asp90His, p.Arg112Gln, p.Gly251Ser, p.Met263Thr, p.Thr295Ile, p.Lys306Thr) and GABRG $_{2}$ (p.Arg82Gln, p.Leu91Ter, p.Arg136Ter, p.Arg177Gly, p.Arg136Ter, p.Leu307Val, p. Lys328Met, p.Arg363Gln, p.Gln390Ter, p.Arg446Trp) [56, 57]. Some of these variations have been associated with epilepsy and episodes of seizures [56, 57]. Likewise, mice models with nonfunctional $\mathrm{GABA}_{\mathrm{B}}$ receptors have spontaneous epilepsy syndromes and behavioral abnormalities [2]. Similarly, SNP R451C in the postsynaptic cell adhesion molecule neuroligin 3 (NL3), which maintains the cross talks between pre- and postsynaptic neuronal cells, has been associated with enhanced GABAergic signaling [58]. In their examination of GDPs, Pizzarelli and Cherubini [58] showed an enhanced depolarizing action of GABA during the first postnatal days in $\mathrm{NL} 3^{\mathrm{R} 451 \mathrm{C}}$ knock-in (KI) mice compared to wild-type mice that led to more frequent GDPs. The mechanism for this phenotype was attributed not to increased availability of $\mathrm{GABA}_{A}$ receptors but to increased presynaptic GABA release and transmission in NL3 ${ }^{\mathrm{R} 451 \mathrm{C}} \mathrm{KI}$ mice [58]. Previously, Tabuchi et al. [59] showed that NL3 ${ }^{\mathrm{R} 451 \mathrm{C}} \mathrm{KI}$ mice showed $\sim 90 \%$ decrease in NL3 and a significant increase in VGAT and gephyrin, both of which facilitate GABA storage and release [60].

The abovementioned studies on genetic regulation of GABAergic neurotransmission provide the understanding of how these mutations in key genes of the GABA pathway contribute to GABAergic dysfunction. However, some cases of mice with null mutations in these key genes have revealed very few associations with developmental abnormalities in the CNS. For instance, GAD mutant mice in some studies showed significantly low normal GABA content but demonstrated no brain defects [2]. Such controversial findings may be rationalized using either the caveat of KO experiments that the germ-line mutations in these mice have developmental compensation that could blur the phenotypic effect or the complexity of these neurological disorders. Although existing evidence suggests a strong inheritance component and genetic causation to these conditions, many of their features and their association with GABAergic neurotransmission dysfunctionality are consistent with epigenetic deregulations. 


\section{Epigenetic Regulations of Postnatal GABAergic Neurotransmission}

Figure 3 depicts various epigenetic regulations of GABAergic genes. It is crucial to analyze the epigenome to better understand the GABAergic neurotransmission regulation and neurological disease etiopathogenesis. Various neurological disease models of mice and patient samples have shed light on the epigenetic marks of GABAergic neurotransmission, including histone modification, chromatin loop formation and DNA methylation. Dysfunction in GABAergic neurotransmission, most likely, may be the outcome of genetic aberration variations of multiple susceptibility gene or epigenetic factors, or synergistic interactions between both.

\section{GABAergic Gene Regulation - Activating H3K4 Methylation and Histone Acetylation}

Histone 3 (H3) methylation at lysine 4 (H3K4) and lysine 27 (H3K27) positions positively and negatively regulates gene expression, respectively, and plays an important role in neural development $[61,62]$. Histone monomethylation (me) or trimethylation (me3) and histone demethylation have been associated with neural development and GABA regulation. A role of H3K4me3 in regulating GABAergic chromatin state and function came to light based on work done in neuropathy mouse models and postmortem prefrontal cortex brain slices from SZ patients $[63,64]$. This dynamic regulation of chromatin structure increases GABAergic gene mRNA levels, including GAD1/Gad1, which affects the synthesis of GABA and maturation of the prefrontal cortex (PFC) in both humans and rodents [63]. GAD1 expression in PFC shows progressive increments throughout prenatal/postnatal development to childhood and peripubertal stage and starts to plateau or slowly decline in later life [63]. This expression dynamic of GAD1 and other GABAergic genes (GAD2/NPY) was shown to directly correlate with the expression of open chromatin mark, $\mathrm{H} 3 \mathrm{~K} 4 \mathrm{me} 3$ and $\mathrm{H} 3 \mathrm{~K} 4 \mathrm{me} 1$, at the corresponding promoters $[63,64]$. In contrast, there is a downregulation of the repressive chromatin marker H3K27me3 at these promoter sites for Gad1, Gad2 and Npy, which is consistent with the well-established finding that there is an inverse correlation between H3K4 methylation and H3K27 methylation in most cell types $[62,65]$. Indeed, the presence of H3K4 methylation marks at a promoter typically makes the site unavailable for H3K27 methylation $[65,66]$.

Alteration in the dynamic process of all three epigenetic regulators - writers, readers and erasers - determines the histone methylation pattern and GABAergic neurotransmission. The epigenetic writer lysine methyltransferase-2A (KMT2A; also known as mixed-lineage leukemia; $M L L, M L L 1$ ), recruited by subunits and transcription factors such as estrogen receptor- $\alpha$, mediates histone lysine methylation (H3K4me3 or H3K4me1) [63, 67-69]. KMT2A binds to both gene promoter and enhancer regions, as well as to unmethylated CpG dinucleotides in order to mediate methylation and chromatin dynamics [69]. KMT2A belongs to trithorax (trx)-related MLL1 family proteins [69]. KMT2A/MIl1 was shown to be predominantly present in the Gad1 promoter in the mouse chromatin extract regulating the histone methylation [69]. On the other hand, mice lacking the wild-type Mll1 allele showed H3K4 trimethylation deficiency and hence a lower expression of the GAD1 gene [63]. iBRAF, a highmobility group-containing protein, can function as a recruiter of Mll1 to the sites of neuronal gene promoters to activate the gene expression [70]. One study showed embryonic lethality and a synaptic plasticity defect in the hippocampus of Mll1 null mice [70]. H3K4 methylation by KMT2A is mediated by the SET1 domain present in the core complex of this epigenetic writer [69]. An additional SET1-related homolog of the MLL family, KMT2B-mediated H3K4 methylation, has also been implicated in maintaining hippocampus-dependent memory 


\section{Epigenetics}

Shrestha and Offer: Epigenetic Regulations of GABAergic Neurotransmission:

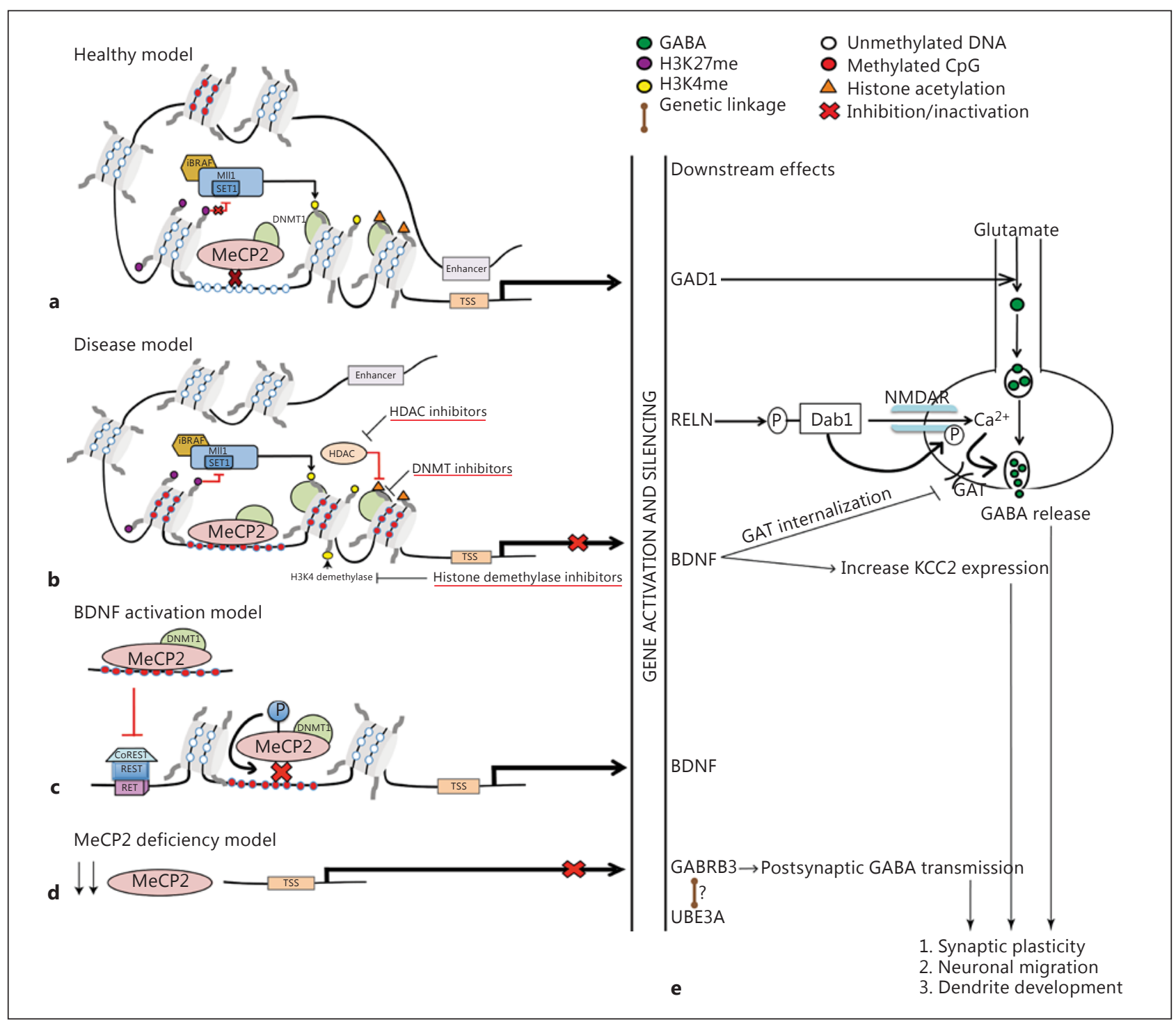

Fig. 3. Epigenetic regulation of postnatal GABAergic neurotransmission. Epigenetic events target various GABAergic genes, including GAD1, RELN, BDNF and GABRB3. a Healthy model of GABAergic neurotransmission. MLL1, with SET domain promotes activating H3K4 methylation; this is enhanced in the presence of GAD1-TSS loop. Decreased expression of DNMT1 in gene promoters reduces MeCP2-mediated GABAergic gene silencing. Additionally, there is a decreased expression of HDAC, preventing the deacetylation of activating histone acetylation marks. There is an overall increase in the transcription and expression of the GAD1, RELN and BDNF genes. b Diseased model of GABAergic neurotransmission. H3K27 methylation blocks the SET domain and inhibits activation of H3K4 methylation. DNMT1 expression and hypermethylation are increased in GABAergic gene promoters in various neurological disorders, such as SZ and Rett's syndrome. This leads to MeCP2-mediated gene silencing of GAD1 and RELN expression. Likewise, BDNF gene expression is also inactivated. c BDNF activation model. Studies also show a BDNF activation model via inactivation of silencing factors REST/CoREST. In some instances, such as when neuronal depolarization occurs, MeCP2 phosphorylation at serine residue causes its release and inhibits its repressive action, leading to BDNF activation. d MeCP2 deficiency model. Unlike in SZ, Rett's syndrome and autism patients show MeCP2 deficiency leading to GABRB3 and UBE3A expression reduction, the mechanism of which is unclear. Some, but not all, studies show genetic linkage between these two genes. e Downstream signaling of GABAergic genes. GAD1 encodes for an important enzyme necessary for GABA synthesis. RELN phosphorylates the Dab1 protein that mediates calcium influx via NMDAR receptor phosphorylation, which in turn enhances GABA release. BDNF protein increases KCC2 expression and inhibits GAT1 internalization, both of which increase GABAergic inhibitory transmission. GABRB3 encodes for the $\mathrm{GABA}_{\mathrm{A}}$ receptor subunit, which mediates GABAergic postsynaptic transmission. All these epigenetic modulations can be used as potential therapeutic targets in the treatment of neurological disorders as indicated by underlined epigenetic drugs including HDAC inhibitors, DNMT inhibitors or demethylase inhibitors in $\mathbf{b}$. 
Shrestha and Offer: Epigenetic Regulations of GABAergic Neurotransmission:

Relevance for Neurological Disorders and Epigenetic Therapy

formation, but the correlation with GABAergic neurotransmission has not been studied [71]. The study by Kim et al. [65] showed that the SET1 domain present in KMT2A is blocked in the presence of H3K27me3 marks, consequently interfering with the H3K4 mono-, di- and trimethylation. The enhancer of zest (EZH)2, which is a component of the polycomb repressive complex 2 (PCR2), is another epigenetic writer that mediates H3K27 methylation [62, 72-74]. EZH2 expression is high in the progenitor cells of the neural cortex, and the expression decreases as the neurogenesis progresses, which is opposite to the expression of GABAergic genes (GAD1/GAD2) [75]. In addition to epigenetic writers, histone demethylases or erasers play an important role in balancing H3K4 and H3K26 methylation. H3K27 demethylase function has been shown to precede H3K4 methylation. Therefore, a decrease in the expression of GABAergic genes by H3K27 methylation can in turn be positively regulated in presence of H3K27 demethylase [65].

Another regulation of $\mathrm{H} 3 \mathrm{~K} 4$ methylation includes chromatin structural regulation. A study showed that the GABAergic gene locus at chromosome 2q31 in human PFC undergoes GAD1 chromatin loop formation during the course of neural differentiation, and H3K4me3 are predominant at the GAD1 transcription start site (TSS) and GAD1-TSS loop as indicated by 3C-CHIP loop assay with H3K4me3 specific antibody [68]. This was shown in tissue homogenates of culture neurons extracted from the frontal cortex and hippocampus [68]. Consequently, the GAD1-TSS chromatin loop allows positioning of upstream enhancer sequence closer to GAD1 TSS with activating HeK4me3 marks that positively regulate GAD1 transcription [68]. Additionally, histone acetylation and deacetylation, with opposing effects, are histone modification marks (writers and erasers) that have been associated with regulation of GABAergic gene expression in SZ and other bipolar disorder [76]. These epigenetic marks have been associated with brain development - brain structural integration as well as memory and cognitive functions and are deregulated in multiple neurological disorders [76, 77]. Histone acetylation, like H3K4 methylation, positively mediates GAD1 transcription [76]. On the other hand, recruitment of histone deacetylase (HDAC) by methyl CpG-binding protein 2 (MeCP2) binding to promoter $\mathrm{CpG}$ in various GABAergic genes acts as transcriptional repression machinery in mouse models, leading to similar phenotypes as seen in Rett's syndrome and ASD [78].

\section{DNA Methylation - DNMT1 and MeCP2-Mediated Transcriptional Silencing}

DNA methyltransferase (DNMT) enzymes, DNMT1, DNMT3A and DNMT3B, are other important epigenetic signaling tools that contribute to changes [65] in neuronal gene expression and GABA regulation (fig. 3). Immunohistochemical and biochemical studies on PFC neurons, pyramidal neurons and glial cells show that GABAergic neurons in PFC have a higher expression of DNMT1 compared to pyramidal neurons and glial cells [79, 80]. Multiple in vitro and in vivo studies suggest the role of DNMT in the epigenetic regulation of important GABAergic gene expression including GAD1 and reelin (RELN); RELN encodes for the reelin protein that is an extracellular matrix protein. Important roles of reelin in neuronal migration, synaptogenesis and axonal branching and dendrite formation have been established [81-83]. Reelin is expressed in varying degrees and regions in the CNS from prenatal to adult stage; in the embryonic stage, reelin protein and mRNA expression localized to Cajal-Retzius cells, a subset of GABAergic neuronal cells, while in the adult brain the localization is prominent in cortical GABAergic neuronal cells [84].

DNMT1 and DNMT3a localization is highly exclusive to GAD1-positive GABAergic neurons $[85,86]$. Experiments looking at the distribution of DNMT1 and DNMT3a using glutamic acid decarboxylase 67-enhanced green fluorescent protein (GAD1-GFP) KI mouse and immuno- 
fluorescence histochemistry analysis showed more than 90\% colocalization of GAD1-GFP with GABAergic neurons in the cortex, striatum and hippocampus [79]. The same study, in line with earlier studies, established that RELN is also expressed in GAD1-GFP-positive GABAergic neurons [79, 87-90]. GABAergic neurons encoding for RELN, similar to GAD1, also have an upregulated expression of DNMT1 mRNA [90]. Additionally, the RELN gene has a CpG-rich promoter region, which makes it susceptible to methylation by DNMTs [91]. Although both DNMT1 and DNMT3a colocalize with GAD1 and are expressed in cortical GABAergic neurons, only increased DMNT1 has been associated with epigenetic hypermethylation of CpG islands in the promoter region of the GAD1 and RELN genes, followed by transcriptional silencing of these genes [79, 80, 92]. As GAD67 synthesize most of GABA, a decrease in GAD1 expression decreases GABA secretion by GABAergic neurons and alters CSF and plasma GABA levels $[90,93]$. A decrease in RELN expression, on the other hand, disrupts dendritic spine development and GABAergic neuronal cell integration during adult neurogenesis [82, 87, 94-96]. Additionally, decreased reelin expressions disturb presynaptic GABA release, which in turn affects GABAergic synaptic plasticity [97]. The mechanism behind this phenomenon is that reelin potently enhances calcium influx through N-methyl-D-aspartate glutamate receptor (NMDAR) by mediating tyrosine phosphorylation of the receptor; consequently, the calcium influx triggers presynaptic GABA release at different neuronal synapses [94, 97-103]. One of the above studies that looked at cytoplasmic adaptor protein Disabled-1 (Dab1) KO mice showed no reelin-mediated phosphorylation and calcium influx demonstrating that phosphorylation of NMDAR receptor is a key step of the mechanism; Dab1 acts downstream of reelin to mediate the phosphorylation [98]. Another important player in the DNMT-regulated hypermethylation is MeCP2, an epigenetic reader, which shows increased affinity to methylated CpG in GAD67 and RELN gene promoters and acts as a transcriptional repressor [104-106]. MeCP2 and HDAC are the components of the repressor complex formed in the DNMT1-methylated site, which is seen in SZ patients or SZ mice models [107].

\section{MeCP2 - Additional Regulations in GABAergic Neurotransmission}

MeCP2 is abundant in neuronal cells, with its expression increasing over the postnatal neurodevelopment $[106,108]$. MeCP2 is expressed $~ 50 \%$ higher in GABAergic neurons compared to non-GABAergic neurons [106]. Studies on MeCP2-deficient mice show the roles of MeCP2 in synaptic plasticity and neuronal connectivity [108]. Medrihan et al. [110], using postnatal MeCP2 KO mice, showed defects in the presynaptic (synaptic vesicles) and postsynaptic (GABA receptors) components of GABAergic synapses leading to depressed GABAergic neurotransmission and excitatory-inhibitory imbalance in MeCP2 deficiency, which disrupt the normal development of GABAergic circuits in the thalamus [109]. Unlike in SZ, this phenotype is seen in patients with Rett's syndrome and ASD. In contrast to Rett's syndrome, MeCP2 deletion in a subset of GABAergic cells (such as in paralvumin-MeCP2-/y mice) disrupts cell plasticity, which affects the cortical plasticity of GABAergic neurons [111]. Some of these GABAergic regulations by MeCP2 are mediated through regulation of the GABA receptor subunit gene, GABA-A receptor, $\beta_{3}$-polypeptide (GABRB3) or other genes affecting the GABAergic neurogenesis or signaling, including BDNF (fig. 3) [106, 108, 109]. GABRB3 encodes for the $\mathrm{GABA}_{\mathrm{A}}$ receptor $\beta_{3}$-subunit, and its downregulation decreases GABA neurotransmission. GABRB3 plays an important role in the sensory processing of cortical circuitry and shows a substantial reduction in expression in Rett's syndrome, ASD and Angelman syndrome (AS) [112]. GABRB3 has two repressor element 1-silencing transcription factor (REST) sites and one MeCP2 binding site (close to REST binding sites) [113]. There is a controversial correlation of MeCP2 expression with GABRB3 expression, which is evident in the 
three neuropathies (Angelman syndrome, ASD and Rett's syndrome); MeCP2 deficiency in these disorders causes a decreased expression of GABRB3 [109, 112, 113]. However, other studies challenge this finding by showing no significant reduction in GABRB3 mRNA and protein levels in brain tissues of MeCP2-deficient mice [114, 115]. LaSalle reasons the time point of MeCP2-deficient mice used or RT-PCR issues to explain the discrepancies observed in these later studies $[105,106,116]$. Some studies have linked the decreased GABRB3 expression in MeCP2 deficiency with decreased expression of the ubiquitin protein ligase E3A (UBE3A) gene [109, 112, 113]. The UBE3A gene, similar to GABRB3, shows a decreased expression in Rett's syndrome and autism, and some familial studies, but not others, have identified linkage between these two genes [112]. Another study implicated the role of MeCP2 in causing the loss of UBE3A antisense gene imprinting, which could not be replicated in later studies $[117,118]$. A clearer understanding of MeCP2 regulations in these genes is needed, with rationally designed investigations into genome-wide screens for novel MeCP2 targets, posttranslational modification and transcription factors.

MeCP2-induced epigenetic regulation of BDNF also influences GABAergic signaling. BDNF is a member of the neurotrophin family and a potent modulator in various aspects of nervous system development. BDNF regulates GABAergic signaling through various mechanisms including reduction of inhibitory actions of GABA transmission by KCC2 upregulation and downregulation of $\mathrm{Cl}^{-}$transport, and increases clearance of synaptic and exsynaptic GABA levels by reducing GAT-1 internalization [119-121]. Li and Pozzo-Miller [122] summarize the MeCP2-dependent modulation of BDNF. The article discusses how MeCP2 can act as a repressive, activating or dual modulation factor of MeCP2 depending on the neuronal activity and other genes/transcripts that come into play. MeCP2 binds to CpG-methylated BDNF promoter sites and inhibits BDNF transcription. In the presence of membrane depolarization neuronal activity, cAMP response element-binding phosphorylation activates BDNF expression, which creates a positive feedback loop via phosphorylation of MeCP2 Ser421 residue causing its release from BDNF promoter in order to activate BDNF transcription [122]. Membrane depolarization also directly causes MeCP2 release by decreasing BDNF promoter methylation [122]. Mecp2 deficiency causes reduction of such neuronal activity and causes downregulation of BDNF expression, which is observed in Rett's syndrome patients [123].

\section{Epigenetic Regulations of GABAergic Neurotransmission in Neurological Disorders - Potential for Epigenetic Therapy}

The recognition of epigenetics as a significant contributor to GABA regulation and their alterations in neurological disorders opens an expanding avenue for pharmacological interventions and drug discoveries (fig. 3b). For instance, alterations in the histone methylation pattern have been associated with the SZ phenotype. Profiling of histone methylation patterns and the mRNA level of the GAD1 promoter in postmortem brain slices of SZ patients showed decreased H3K4 trimethylation and a decreased GAD1 mRNA level, but increased H3K27me3 [63]. This result was attributed to SNPs located in proximity to the GAD1 TSS site that disrupts the chromatin loop formation necessary to increase GAD1 mRNA expression $[63,68]$. The samples from SZ patients treated with an atypical antipsychotic clozapine showed a reversed phenotype with increased H3K4me3 marks and GAD1 mRNA expression attributed to increased expression of MLL1 [63]. In these patients, histone methyltransferase specific to active histone marks (H3K4), inhibitor of EZH2 (H3K27 methyltransferase) or histone demethylase inhibitors may have potential therapeutic benefits [124]. For instance, GSK126 and EPZ6438, used as therapy candidates for lymphoma, which compete with S-adenosyl- 
methionine, inhibit EZH2 activity $[125,126]$. In addition, it also decreases global H3K27 methylation; therefore, it is important to explore its therapeutic potential in GABA-related neurological disorders. Lysine-specific demethylase-1 (LSD-1) inhibitor, which increases H3K4 methylation and has been used in the treatment of colon cancer, may be a promising epigenetic therapy to prevent decreased GAD1 expression seen in these neurological disorders [124].

Likewise, an analysis of neuronal tissues of the PFC - hippocampus and medial temporal lobe - from patients with SZ and a subset of with bipolar disorder showed increased HDAC1 expression, resulting in the loss of histone acetylation in the promoter of GAD1 and other SZ genes in these regions [76]. 'Decreased prepulse inhibition', an important phenotype in SZ patients and animal models, and decreased GAD1 transcripts caused by overexpression of HDAC1 in adult mouse hippocampus confirm the finding that histone acetylation is associated with GABAergic neurotransmission and SZ phenotype [76, 77]. Unsurprisingly, there are studies focusing on HDAC inhibitors, reversing the effect of HDAC1 increased expression, in various neurological disorders [77]. Studies focusing on various HDAC inhibitors such as valproate and clozapine have shown that they improve cognitive functions and synaptic plasticity in SZ mouse models $[77,127]$. A study elucidating the mechanism of hyposensitivity to GABAergic neurotransmission during alcohol withdrawal showed that increased levels of HDAC transcripts in the alcohol withdrawal brain slices reduced the level of $\alpha_{1}$-subunit of $\mathrm{GABA}_{\mathrm{A}}$ receptors. This phenomenon is relieved in the presence of HDAC-inhibitors [128]. A similar phenomenon may come into play in some of these neurological disorders. However, these HDAC inhibitors only target about $10 \%$ of the acetylation site and also have off-target effects, so more work is necessary to understand and improve the target specificity and isoform-specific HDAC inhibitors.

Increased DNMT1 and MeCP2-mediated hypermethylation and silencing of GAD67 and RELN has been implicated in various neuropathies, including bipolar disorder, depressionlike behavior, autism and particularly in SZ $[26,79,96]$. Mouse models of these neurological disorders and patient samples show increased transcripts of DNMT1; consequently, there is transcriptional silencing of GABAergic genes (GAD1, RELN, BDNF) leading to neuropathy phenotypes $[26,79,96]$. Pertaining to various modes of regulation and association with neuropathies of DNMT1 and MeCP2 that promotes hypermethylation and transcriptional silencing, DNMT inhibitors or demethylating agents have been under preclinical and clinical investigations as a therapeutic strategy in various disorders. In vivo and in vitro experiments on such DNMT inhibitors show a reduction in total DNMT enzyme activity and reduction in the DNA hypermethylation in the RELN and GAD1 gene promoters, hence normalizing the GABAergic neurotransmission [107]. The most commonly studied compounds are azacitidine and decitabine, which form an irreversible complex with DNMT1 and inhibit CpG methylation $[129,130]$. These inhibitors have been used in cancer cells in vivo and in vitro to downregulate cell division, but the effects are questionable in postmitotic differentiated neurons. Another drug, procainamide, used in the treatment of cardiac arrhythmia, has however shown some promise in preventing hypermethylation of RELN and GAD1 in differentiated neuronal cells in vivo in mice models [130]. HDAC inhibitors (e.g. VPA and MS-275) have also been shown to promote GAD1 and RELN promoter demethylation by inhibiting DNMT activity [130]. MeCP2 has, however, proved to be a complicated target for therapy because of the controversial findings in MeCP2-deficient mice (i.e. decreased expression of GABA receptors, as well as some brain developmental abnormalities) [106, 108, 109, 131]. As discussed by Castro et al. [131], MeCP2 control of BDNF, observed in Rett's syndrome, is very complex, and interacts with various binding sites, binding partners and signaling pathways. Combination therapy may be more applicable in the disorders (Rett's syndrome, ASD, PWS) with MeCP2 regulations. 
It is important to note that many of these epigenetic therapies are being used or studied as potential therapy in various forms of cancer. For instance, the association of overexpression of EZH2 with poor prognosis in EZH2 mutant non-Hodgkin lymphoma has made GSK126 and EPZ6438 potential candidates for lymphoma therapy $[125,126]$. Similarly, valproic acid/ valproate, clozapine, azacitidine and decitabine have been shown to have an anticancer effect. Multi-effects of these drugs can, however, have a positive benefit or adverse effects. For instance, one study, although inconclusive, looked at a possible cancer-protective effect of valproate in people with epilepsy being treated with this drug [132]. On the other hand, most of these drugs, including azacitidine and decitabine, cause neurotoxic side effects, which might possibly be associated with GABA function. There is a need for more clinical studies looking at the correlations of anticancer effect and neurotoxicity, as well as the role of GABAergic neurotransmission alteration as a possible mechanism of neurotoxicity as a result of these drugs.

\section{Conclusions}

This review has shed light on: (1) the complexity of GABAergic neurotransmission and its development, (2) the control of brain development from early neuronal sculpting to adult neurogenesis conferred by GABAergic neurotransmission, (3) the transcriptional regulation of GABAergic neurons in prenatal state, and more comprehensively (4) important epigenetic regulatory marks of GABAergic neurotransmission $[26,42,133]$. The paper then discussed the potentials of epigenetic marks as the therapeutic targets in the treatment of neurological disorders $[134,135]$. Due to its conserved nature and wide range of functions during neurogenesis, it is not surprising that the GABA neurotransmission is highly regulated, genetically and epigenetically, during the development as well as the postnatal stage. GABAergic neuron formation and migration are mediated by different sets of transcription factors in certain brain regions, including the Dlx family, TAL1/2, GATA and Pfta1 [42]. Additionally, in the postnatal stage of brain development, there are various epigenetic modulations involved in GABAergic neurotransmission such as DNMT-mediated methylation that causes transcriptional silencing and histone modification marks that increase or decrease gene expressions. Studies on neurological disease mice models and SZ, ASD and Rett's syndrome patients show disruptions in these epigenetic modifications, including an increased expression of the gene silencing epigenetic mark H3K27me, mutations in MeCP2 or a blockade of the SET domain in MeCP2. These deregulations result in a decreased expression of important GABAergic genes, including GAD1, BDNF and RELN, and cause disturbance of normal GABAergic neurotransmission and normal brain development, including synaptic plasticity and neuronal integrations.

Given the complex nature of neurological disorders, the mode of inheritance or genetic modulation of GABAergic neurotransmission only provides an incomplete explanation of the role of GABA in the pathogenicity of these diseases. Therefore, neuroepigenetics is an important and evolving field, as we are beginning to better understand the epigenomic markers that confer reversible and bidirectional regulation of various genes that play a role in the GABAergic pathway. Understanding the epigenetic regulations of GABAergic neurotransmission can allow us to explore possible therapeutic targets for GABA-associated neurological disorders. For instance, HDAC inhibitors and DNMT inhibitors have proven to reverse various symptoms associated with disruption in GABAergic neurotransmission caused by histone modification and DNA hypermethylation. Some DNMT inhibitors have been a major attraction in multiple preclinical and clinical trials. It will be important to clarify, for each disorder and associated phenotype, which of the affected genes or genomic loci are important 
for the alteration in GABAergic neurotransmission and disease manifestation, versus which are merely bystanders and irrelevant to the disease process. Additionally, further elucidation is important in terms of the causal relationship among epigenetic marks, GABA neurotransmission and neurological disorders. In other words, it is unclear whether the epigenetic modification precedes GABA dysfunction causing neuropathies or vice versa, or if there is a positive/negative feedback loop in the process. When studying epigenetic therapy for these neurological disorders, it is also important to consider the multi-effects of these available drugs, most of which demonstrate anticancer function. Physicians must be aware of any neurotoxicity side effects the drug might cause due to its effect on GABA function, or any possible drug-drug interaction with other chemo-regimens. It is important to explore the variation of the effect these drugs might have in patients with a combination of disease (cancer and neurodisorders) versus single disease states. Hence, in the future, combining the current knowledge of GABAergic neurotransmission epigenetic regulation and its association with neurological disorders, with improved understanding of the abovementioned questions, will significantly facilitate the development of more effective and individualized therapies.

\section{References}

1 Lujan R, Shigemoto R, Lopez-Bendito G: Glutamate and GABA receptor signalling in the developing brain. Neuroscience 2005;130;567-580.

-2 Owens DF, Kriegstein AR: Is there more to GABA than synaptic inhibition? Nat Rev Neurosci 2002;3:715-727.

3 Olsen RW: GABA; in Charney D, Davis KL, Coyle JT, Nemeroff C (eds): Neuropsychopharmacology. Philadelphia, Lippincott, Williams \& Wilkins, 2002.

4 Bouche N, Lacombe B, Fromm H: GABA signaling: a conserved and ubiquitous mechanism. Trends Cell Biol 2003;13:603-606.

5 Chen Y, Dong E, Grayson DR: Analysis of the GAD1 promoter: trans-acting factors and DNA methylation converge on the $5^{\prime}$ untranslated region. Neuropharmacology 2011;60:1075-1087.

-6 Markwardt S, Overstreet-Wadiche L: GABAergic signalling to adult-generated neurons. J Physiol 2008;586: 3745-3749.

7 Li Y, Xiang YY, Lu WY, Liu C, Li J: A novel role of intestine epithelial GABAergic signaling in regulating intestinal fluid secretion. Am J Physiol Gastrointest Liver Physiol 2012;303:G453-G460.

-8 Spitzer NC: How GABA generates depolarization. J Physiol 2010;588:757-758.

$\checkmark 9 \mathrm{He}$ Q Nomura T, Xu J, Contractor A: The developmental switch in GABA polarity is delayed in fragile X mice. J Neurosci 2014;34:446-450.

10 Tyzio R, Holmes GL, Ben-Ari Y, Khazipov R: Timing of the developmental switch in GABA(A) mediated signaling from excitation to inhibition in CA3 rat hippocampus using gramicidin perforated patch and extracellular recordings. Epilepsia 2007;48(suppl 5):96-105.

11 Hasbargen T, et al: Role of NKCC1 and KCC2 in the development of chronic neuropathic pain following spinal cord injury. Ann N Y Acad Sci 2010;1198:168-172.

12 Scimemi A: Structure, function, and plasticity of GABA transporters. Front Cell Neurosci 2014;8:161.

13 Roberts E, Rothstein M, Baxter CF: Some metabolic studies of gamma-aminobutyric acid. Proc Soc Exp Biol Med 1958;97:796-802.

14 Bowery NG, Smart TG: GABA and glycine as neurotransmitters: a brief history. Br J Pharmacol 2006;147(suppl 1):S109-S119.

15 McCormick DA: GABA as inhibitory neurotransmitter in human cerebral cortex. J Neurophysiol 1989;62: 1018-1028.

16 Dichter MA: Physiological identification of GABA as the inhibitory transmitter for mammalian cortical neurons in cell culture. Brain Res 1980;190:111-121.

$\checkmark 17$ Faingold CL, Gehlbach G, Caspary DM: On the role of GABA as an inhibitory neurotransmitter in inferior colliculus neurons: iontophoretic studies. Brain Res 1989;500:302-312.

-18 Eyzaguirre C, Kuffler SW: Processes of excitation in the dendrites and in the soma of single isolated sensory nerve cells of the lobster and crayfish. J Gen Physiol 1955;39:87-119.

19 Kuffler SW, Edwards C: Mechanism of gamma aminobutyric acid (GABA) action and its relation to synaptic inhibition. J Neurophysiol 1958;21:589-610.

20 McIntire SL, Jorgensen E, Kaplan J, Horvitz HR: The GABAergic nervous system of Caenorhabditis elegans. Nature 1993;364:337-341.

21 Leinekugel X, et al: GABA is the principal fast-acting excitatory transmitter in the neonatal brain. Adv Neurol 1999;79:189-201. 
22 Chen G, Trombley PQ, van den Pol AN: Excitatory actions of GABA in developing rat hypothalamic neurones. J Physiol 1996;494(Pt 2):451-464.

23 Ben-Ari Y, Gaiarsa JL, Tyzio R, Khazipov R: GABA: a pioneer transmitter that excites immature neurons and generates primitive oscillations. Physiol Rev 2007;87:1215-1284.

24 Ben-Ari Y: Excitatory actions of GABA during development: the nature of the nurture. Nat Rev Neurosci 2002; 3:728-739.

25 Ganguly K, et al: GABA itself promotes the developmental switch of neuronal GABAergic reponses from excitation to inhibition. Cell 2001;105:521-532.

26 Schmidt MJ, Mirnics K: Neurodevelopment, GABA system dysfunction, and schizophrenia. Neuropsychopharmacology 2015;40:190-206.

-27 Sipila ST, Huttu K, Soltesz I, Voipio J, Kaila K: Depolarizing GABA acts on intrinsically bursting pyramidal neurons to drive giant depolarizing potentials in the immature hippocampus. J Neurosci 2005;25:5280-5289.

28 Tozuka Y, Fukuda S, Namba T, Seki T, Hisatsune T: GABAergic excitation promotes neuronal differentiation in adult hippocampal progenitor cells. Neuron 2005;47:803-815.

29 Le Magueresse C, Monyer H: GABAergic interneurons shape the functional maturation of the cortex. Neuron 2013;77:388-405.

30 Giachino C, et al: GABA suppresses neurogenesis in the adult hippocampus through GABAB receptors. Development 2014;141:83-90.

-31 Andang M, et al: Histone H2AX-dependent GABA(A) receptor regulation of stem cell proliferation. Nature 2008;451:460-464.

32 Luhmann HJ, Fukuda A, Kilb W: Control of cortical neuronal migration by glutamate and GABA. Front Cell Neurosci 2015;9:4.

-33 Represa A, Ben-Ari Y: Trophic actions of GABA on neuronal development. Trends Neurosci 2005;28:278-283.

-34 Wang DD, Kriegstein AR: Defining the role of GABA in cortical development. J Physiol 2009;587:1873-1879.

35 Ge S, et al: GABA regulates synaptic integration of newly generated neurons in the adult brain. Nature 2006; 439:589-593.

-36 Wang DD, Kriegstein AR: GABA regulates excitatory synapse formation in the neocortex via NMDA receptor activation. J Neurosci 2008;28:5547-5558.

-37 Pizzarelli R, Cherubini E: Alterations of GABAergic signaling in autism spectrum disorders. Neural Plast 2011; 2011:297153.

38 Rubenstein JL, Merzenich MM: Model of autism: increased ratio of excitation inhibition in key neural systems. Gene Brain Behav 2003;2:255-267.

-39 Han B, Bellemer A, Koelle MR: An evolutionarily conserved switch in response to GABA affects development and behavior of the locomotor circuit of Caenorhabditis elegans. Genetics 2015;199:1159-1172.

-40 Deidda G, et al: Early depolarizing GABA controls critical-period plasticity in the rat visual cortex. Nat Neurosci 2015;18:87-96.

41 Rossignol E: Genetics and function of neocortical GABAergic interneurons in neurodevelopmental disorders. Neural Plast 2011;2011:649325.

42 Achim K, Salminen M, Partanen J: Mechanisms regulating GABAergic neuron development. Cell Mol Life Sci 2014;71:1395-1415.

43 Lahti L, Achim K, Partanen J: Molecular regulation of GABAergic neuron differentiation and diversity in the developing midbrain. Acta Physiol (Oxf) 2013;207:616-627.

44 Sultan KT, Brown KN, Shi SH: Production and organization of neocortical interneurons. Front Cell Neurosci 2013; 7:221.

45 Maira M, Long JE, Lee AY, Rubenstein JL, Stifani S: Role for TGF-beta superfamily signaling in telencephalic GABAergic neuron development. J Neurodev Disord 2010;2:48-60.

-46 Alcantara S, Pozas E, Ibanez CF, Soriano E: BDNF-modulated spatial organization of Cajal-Retzius and GABAergic neurons in the marginal zone plays a role in the development of cortical organization. Cereb Cortex 2006;16:487-499.

47 MacDonald RB, et al: The ascl1a and dlx genes have a regulatory role in the development of GABAergic interneurons in the zebrafish diencephalon. Dev Biol 2013;381:276-285.

48 Hamilton SP, et al: Analysis of four DLX homeobox genes in autistic probands. BMC Genet 2005;6:52.

-49 Chang SC, Pauls DL, Lange C, Sasanfar R, Santangelo SL: Common genetic variation in the GAD1 gene and the entire family of DLX homeobox genes and autism spectrum disorders. Am J Med Genet B Neuropsychiatr Genet 2011;156:233-239.

50 Krey JF, Dolmetsch RE: Molecular mechanisms of autism: a possible role for $\mathrm{Ca}^{2+}$ signaling. Curr Opin Neurobiol 2007;17:112-119.

-51 Benes FM, Berretta S: GABAergic interneurons: implications for understanding schizophrenia and bipolar disorder. Neuropsychopharmacology 2001;25:1-27.

52 Hardt 0, et al: Gene expression analysis defines differences between region-specific GABAergic neurons. Mol Cell Neurosci 2008;39:418-428.

53 Du J, et al: Comprehensive analysis of polymorphisms throughout GAD1 gene: a family-based association study in schizophrenia. J Neural Transm 2008;115:513-519.

54 Macdonald RL, Kang JQ, Gallagher MJ: Mutations in GABAA receptor subunits associated with genetic epilepsies. J Physiol 2010;588:1861-1869. 
Shrestha and Offer: Epigenetic Regulations of GABAergic Neurotransmission:

Relevance for Neurological Disorders and Epigenetic Therapy

55 Baulac S, et al: First genetic evidence of GABA(A) receptor dysfunction in epilepsy: a mutation in the gamma2subunit gene. Nat Genet 2001;28:46-48.

56 Database of Single Nucleotide Polymorphisms (dbSNP). Bethesda, National Center for Biotechnology Information. http://www.ncbi.nlm.nih.gov/SNP/.

57 Landrum MJ, et al: ClinVar: public archive of interpretations of clinically relevant variants. Nucleic Acids Res 2016;44:D862-D866.

58 Pizzarelli R, Cherubini E: Developmental regulation of GABAergic signalling in the hippocampus of neuroligin 3 R451C knock-in mice: an animal model of autism. Front Cell Neurosci 2013;7:85.

59 Tabuchi K, Blundell J, Etherton MR, Hammer RE, Liu X, Powell CM, Südhof TC: A neuroligin-3 mutation implicated in autism increases inhibitory synaptic transmission in mice. Science 2007;318:71-76.

60 Tyagarajan SK, Fritschy JM: Gephyrin: a master regulator of neuronal function? Nat Rev Neurosci 2014;15: 141-156.

61 Lee S, Lee SK: Crucial roles of histone-modifying enzymes in mediating neural cell-type specification. Curr Opin Neurobiol 2010;20:29-36.

62 Cui P, et al: Comparative analyses of H3K4 and H3K27 trimethylations between the mouse cerebrum and testis. Genomics Proteomics Bioinformatics 2012;10:82-93.

63 Huang HS, et al: Prefrontal dysfunction in schizophrenia involves mixed-lineage leukemia 1-regulated histone methylation at GABAergic gene promoters. J Neurosci 2007;27:11254-11262.

-64 Peter CJ, Akbarian S: Balancing histone methylation activities in psychiatric disorders. Trends Mol Med 2011; 17:372-379.

65 Kim DH, et al: Histone H3K27 trimethylation inhibits H3 binding and function of SET1-like H3K4 methyltransferase complexes. Mol Cell Biol 2013;33:4936-4946.

$66 \mathrm{Xu} \mathrm{J}$, Andreassi M: Reversible histone methylation regulates brain gene expression and behavior. Horm Behav 2011;59:383-392.

67 Black JC, Whetstine JR: Tipping the lysine methylation balance in disease. Biopolymers 2013;99:127-135.

68 Bharadwaj R, et al: Conserved chromosome 2q31 conformations are associated with transcriptional regulation of GAD1 GABA synthesis enzyme and altered in prefrontal cortex of subjects with schizophrenia. J Neurosci 2013;33:11839-11851.

69 Rao RC, Dou Y: Hijacked in cancer: the KMT2 (MLL) family of methyltransferases. Nat Rev Cancer 2015;15: 334-346.

-70 Akbarian S, Huang HS: Epigenetic regulation in human brain-focus on histone lysine methylation. Biol Psychiatry 2009;65:198-203.

71 Kerimoglu C, et al: Histone-methyltransferase MLL2 (KMT2B) is required for memory formation in mice. J Neurosci 2013;33:3452-3464.

72 Xiang Y, et al: JMJD3 is a histone H3K27 demethylase. Cell Res 2007;17:850-857.

73 Estaras C, et al: Genome-wide analysis reveals that Smad3 and JMJD3 HDM co-activate the neural developmental program. Development 2012;139:2681-2691.

74 Burgold T, et al: The histone H3 lysine 27-specific demethylase Jmjd3 is required for neural commitment. PLoS One 2008;3:e3034.

-75 Pereira JD, et al: Ezh2, the histone methyltransferase of PRC2, regulates the balance between self-renewal and differentiation in the cerebral cortex. Proc Natl Acad Sci USA 2010;107:15957-15962.

-76 Hasan A, Mitchell A, Schneider A, Halene T, Akbarian S: Epigenetic dysregulation in schizophrenia: molecular and clinical aspects of histone deacetylase inhibitors. Eur Arch Psychiatry Clin Neurosci 2013;263:273-284.

77 Volmar C-H, et al: Histone deacetylases (HDACs) and brain function. Neuroepigenetics 2015;1:20-27.

-78 Abel T, Zukin RS: Epigenetic targets of HDAC inhibition in neurodegenerative and psychiatric disorders. Curr Opin Pharmacol 2008;8:57-64

79 Kadriu B, Guidotti A, Chen Y, Grayson DR: DNA methyltransferases1 (DNMT1) and 3a (DNMT3a) colocalize with GAD67-positive neurons in the GAD67-GFP mouse brain. J Compar Neurol 2012;520:1951-1964.

$>80$ Grayson DR, et al: The human reelin gene: transcription factors (+), repressors $(-)$ and the methylation switch (+/-) in schizophrenia. Pharmacol Ther 2006;111:272-286.

81 Lakatosova S, Ostatnikova D: Reelin and its complex involvement in brain development and function. Int J Biochem Cell Biol 2012;44:1501-1504.

82 Kingman II: Reelin in brain development studies. Drug Discov Today 2000;5:42.

83 Tissir F, Goffinet AM: Reelin and brain development. Nat Rev Neurosci 2003;4:496-505.

-84 Caruncho HJ, Dopeso-Reyes IG, Loza MI, Rodriguez MA: A GABA, reelin, and the neurodevelopmental hypothesis of schizophrenia. Crit Rev Neurobiol 2004;16:25-32.

85 Zhubi A, et al: An upregulation of DNA-methyltransferase 1 and 3a expressed in telencephalic GABAergic neurons of schizophrenia patients is also detected in peripheral blood lymphocytes. Schizophr Res 2009;111: $115-122$.

86 Veldic M, et al: DNA-methyltransferase 1 mRNA is selectively overexpressed in telencephalic GABAergic interneurons of schizophrenia brains. Proc Natl Acad Sci USA 2004;101:348-353.

-87 Pappas GD, Kriho V, Pesold C: Reelin in the extracellular matrix and dendritic spines of the cortex and hippocampus: a comparison between wild type and heterozygous reeler mice by immunoelectron microscopy. J Neurocytol 2001;30:413-425. 
Shrestha and Offer: Epigenetic Regulations of GABAergic Neurotransmission:

Relevance for Neurological Disorders and Epigenetic Therapy

88 Pesold C, et al: Reelin is preferentially expressed in neurons synthesizing gamma-aminobutyric acid in cortex and hippocampus of adult rats. Proc Natl Acad Sci USA 1998;95:3221-3226.

89 Pesold C, Liu WS, Guidotti A, Costa E, Caruncho HJ: Cortical bitufted, horizontal, and Martinotti cells preferentially express and secrete reelin into perineuronal nets, nonsynaptically modulating gene expression. Proc Natl Acad Sci USA 1999;96:3217-3222.

-90 Kundakovic M, Chen Y, Costa E, Grayson DR: DNA methyltransferase inhibitors coordinately induce expression of the human reelin and glutamic acid decarboxylase 67 genes. Mol Pharmacol 2007;71:644-653.

91 Fatemi SH: Reelin glycoprotein: structure, biology and roles in health and disease. Mol Psychiatry 2005;10: 251-257.

92 Veldic M, Guidotti A, Maloku E, Davis JM, Costa E: In psychosis, cortical interneurons overexpress DNA-methyltransferase 1. Proc Natl Acad Sci USA 2005;102:2152-2157.

-93 Fatemi SH, Stary JM, Earle JA, Araghi-Niknam M, Eagan E: GABAergic dysfunction in schizophrenia and mood disorders as reflected by decreased levels of glutamic acid decarboxylase 65 and $67 \mathrm{kDa}$ and Reelin proteins in cerebellum. Schizophr Res 2005;72:109-122.

94 Campo CG, Sinagra M, Verrier D, Manzoni OJ, Chavis P: Reelin secreted by GABAergic neurons regulates glutamate receptor homeostasis. PLoS One 2009;4:e5505.

$\checkmark 95$ Tueting P, Doueiri MS, Guidotti A, Davis JM, Costa E: Reelin down-regulation in mice and psychosis endophenotypes. Neurosci Biobehav Rev 2006;30:1065-1077.

-96 Fenton EY, et al: Imipramine protects against the deleterious effects of chronic corticosterone on depressionlike behavior, hippocampal reelin expression, and neuronal maturation. Prog Neuropsychopharmacol Biol Psychiatry 2015;60:52-59.

97 Halabisky B, Friedman D, Radojicic M, Strowbridge BW: Calcium influx through NMDA receptors directly evokes GABA release in olfactory bulb granule cells. J Neurosci 2000;20:5124-5134.

98 Chen Y, et al: Reelin modulates NMDA receptor activity in cortical neurons. J Neurosci 2005;25:8209-8216.

-99 Sinagra M, et al: Reelin, very-low-density lipoprotein receptor, and apolipoprotein E receptor 2 control somatic NMDA receptor composition during hippocampal maturation in vitro. J Neurosci 2005;25:61276136.

100 Buret L, van den Buuse M: Corticosterone treatment during adolescence induces down-regulation of reelin and NMDA receptor subunit GLUN2C expression only in male mice: implications for schizophrenia. Int J Neuropsychopharmacol 2014;17:1221-1232.

101 Qiu S, Zhao LF, Korwek KM, Weeber EJ: Differential reelin-induced enhancement of NMDA and AMPA receptor activity in the adult hippocampus. J Neurosci 2006;26:12943-12955.

102 Glitsch MD: Calcium influx through N-methyl-D-aspartate receptors triggers GABA release at interneuronPurkinje cell synapse in rat cerebellum. Neuroscience 2008;151:403-409.

103 Kavalali ET: The mechanisms and functions of spontaneous neurotransmitter release. Nat Rev Neurosci 2015; 16:5-16.

104 Sun W, Zang L, Shu Q, Li X: From development to diseases: the role of 5hmC in brain. Genomics 2014;104: 347-351.

105 Zhubi A, et al: Increased binding of MeCP2 to the GAD1 and RELN promoters may be mediated by an enrichment of 5-hmC in autism spectrum disorder (ASD) cerebellum. Transl Psychiatry 2014;4:e349.

106 Chao HT, et al: Dysfunction in GABA signalling mediates autism-like stereotypies and Rett syndrome phenotypes. Nature 2010;468:263-269.

107 Grayson DR, Kundakovic M, Chen Y, Dong E, Guidotti A: Epigenetic regulation of GABAergic targets in psychiatry; in Petronis A, Mill J (eds): Brain, Behavior and Epigenetics. Berlin, Springer, 2011, pp 23-40.

108 Gonzales ML, LaSalle JM: The role of MeCP2 in brain development and neurodevelopmental disorders. Curr Psychiatry Rep 2010;12:127-134.

109 Zhang ZW, Zak JD, Liu H: MeCP2 is required for normal development of GABAergic circuits in the thalamus. J Neurophysiol 2010;103:2470-2481.

110 Medrihan L, et al: Early defects of GABAergic synapses in the brain stem of a MeCP2 mouse model of Rett syndrome. J Neurophysiol 2008;99:112-121.

$\checkmark 111 \mathrm{He}$ LJ, et al: Conditional deletion of Mecp2 in parvalbumin-expressing GABAergic cells results in the absence of critical period plasticity. Nat Commun 2014;5:5036.

112 Samaco RC, Hogart A, LaSalle JM: Epigenetic overlap in autism-spectrum neurodevelopmental disorders: MECP2 deficiency causes reduced expression of UBE3A and GABRB3. Hum Mol Genet 2005;14:483-492.

113 Tanaka M, DeLorey TM, Delgado-Escueta A, Olsen RW: GABRB3, epilepsy, and neurodevelopment; in Noebels JL, et al (eds): Jasper's Basic Mechanisms of the Epilepsies, ed 4. Bethesda, National Center for Biotechnology Information, 2012.

114 Jordan C, Francke U: Ube3a expression is not altered in Mecp2 mutant mice. Hum Mol Genet 2006;15:2210 2215.

115 Lawson-Yuen A, et al: Ube3a mRNA and protein expression are not decreased in Mecp2R168X mutant mice. Brain Res 2007;1180:1-6.

116 LaSalle JM: The odyssey of MeCP2 and parental imprinting. Epigenetics 2007;2:5-10.

$\checkmark 117$ Makedonski K, Abuhatzira L, Kaufman Y, Razin A, Shemer R: MeCP2 deficiency in Rett syndrome causes epigenetic aberrations at the PWS/AS imprinting center that affects UBE3A expression. Hum Mol Genet 2005; 14:1049-1058 
Shrestha and Offer: Epigenetic Regulations of GABAergic Neurotransmission:

Relevance for Neurological Disorders and Epigenetic Therapy

118 Francke U: Mechanisms of disease: neurogenetics of MeCP2 deficiency. Nature clinical practice. Neurology 2006;2:212-221.

119 Wardle RA, Poo MM: Brain-derived neurotrophic factor modulation of GABAergic synapses by postsynaptic regulation of chloride transport. J Neurosci 2003;23:8722-8732.

120 Vaz SH, et al: Brain-derived neurotrophic factor (BDNF) enhances GABA transport by modulating the trafficking of GABA transporter-1 (GAT-1) from the plasma membrane of rat cortical astrocytes. J Biol Chem 2011; 286:40464-40476.

121 Puskarjov M, et al: BDNF is required for seizure-induced but not developmental up-regulation of KCC2 in the neonatal hippocampus. Neuropharmacology 2015;88:103-109.

122 Li W, Pozzo-Miller L: BDNF deregulation in Rett syndrome. Neuropharmacology 2014;76(Pt C):737-746.

-123 Xu X, Kozikowski AP, Pozzo-Miller L: A selective histone deacetylase-6 inhibitor improves BDNF trafficking in hippocampal neurons from Mecp2 knockout mice: implications for Rett syndrome. Front Cell Neurosci 2014; 8:68.

124 Kelly TK, De Carvalho DD, Jones PA: Epigenetic modifications as therapeutic targets. Nat Biotechnol 2010;28: 1069-1078.

125 Knutson SK, et al: Selective inhibition of EZH2 by EPZ-6438 leads to potent antitumor activity in EZH2-mutant non-Hodgkin lymphoma. Mol Cancer Ther 2014;13:842-854.

126 McCabe MT, et al: EZH2 inhibition as a therapeutic strategy for lymphoma with EZH2-activating mutations. Nature 2012;492:108-112.

127 Cha DS, Kudlow PA, Baskaran A, Mansur RB, McIntyre RS: Implications of epigenetic modulation for novel treatment approaches in patients with schizophrenia. Neuropharmacology 2014;77:481-486.

$\$ 128$ Arora DS, et al: Hyposensitivity to gamma-aminobutyric acid in the ventral tegmental area during alcohol withdrawal: reversal by histone deacetylase inhibitors. Neuropsychopharmacology 2013;38:1674-1684.

$\checkmark 129$ Xu Z, Li H, Jin P: Epigenetics-based therapeutics for neurodegenerative disorders. Curr Transl Geriatr Exp Gerontol Rep 2012;1:229-236.

130 Costa E, et al: GABAergic promoter hypermethylation as a model to study the neurochemistry of schizophrenia vulnerability. Expert Rev Neurother 2009;9:87-98.

131 Castro J, Mellios N, Sur M: Mechanisms and therapeutic challenges in autism spectrum disorders: insights from Rett syndrome. Curr Opin Neurol 2013;26:154-159.

132 Singh G, et al: Cancer risk in people with epilepsy using valproate-sodium. Acta Neurol Scand 2012;125:234240.

$\$ 133$ Feng J, Fouse S, Fan G: Epigenetic regulation of neural gene expression and neuronal function. Pediatr Res 2007;61:58R-63R.

134 Cellot G, Cherubini E: GABAergic signaling as therapeutic target for autism spectrum disorders. Front Pediatr 2014;2:70.

135 Tsankova N, Renthal W, Kumar A, Nestler EJ: Epigenetic regulation in psychiatric disorders. Nat Rev Neurosci 2007;8:355-367. 